\title{
Analysis of pork adulteration in beef meatball using Fourier transform infrared (FTIR) spectroscopy.
}

\begin{abstract}
Meatball is one of the favorite foods in Indonesia. The adulteration of pork in beef meatball is frequently occurring. This study was aimed to develop a fast and non destructive technique for the detection and quantification of pork in beef meatball using Fourier transform infrared (FTIR) spectroscopy and partial least square (PLS) calibration. The spectral bands associated with pork fat (PF), beef fat (BF), and their mixtures in meatball formulation were scanned, interpreted, and identified by relating them to those spectroscopically representative to pure $\mathrm{PF}$ and BF. For quantitative analysis, PLS regression was used to develop a calibration model at the selected fingerprint regions of $1200-1000 \mathrm{~cm}-1$. The equation obtained for the relationship between actual PF value and FTIR predicted values in PLS calibration model was $y=0.999 x+0.004$, with coefficient of determination (R2) and root mean square error of calibration are 0.999 and 0.442 , respectively. The PLS calibration model was subsequently used for the prediction of independent samples using laboratory made meatball samples containing the mixtures of $\mathrm{BF}$ and $\mathrm{PF}$. Using 4 principal components, root mean square error of prediction is 0.742 . The results showed that FTIR spectroscopy can be used for the detection and quantification of pork in beef meatball formulation for Halal verification purposes.
\end{abstract}

Keyword: Adulteration; Beef meatball; FTIR spectroscopy; Partial least square (PLS); Pork. 